\title{
First report of Kingella kingae diagnosed in pediatric bone and joint infections in Morocco
}

\author{
Kaoutar Moutaouakkil ${ }^{1}$, Bouchra Oumokhtar ${ }^{1 *}$, Hicham Abdellaoui ${ }^{2}$, Samira El Fakir ${ }^{3}$, Btissam Arhoune ${ }^{1}$, \\ Mustapha Mahmoud ${ }^{4}$, Karima Atarraf ${ }^{2}$ and Moulay Abderrahmane Afifi $^{2}$
}

\begin{abstract}
Background: The progress of diagnostic strategies and molecular methods improved the detection of Kingella kingae in bone and joint infections, and now, Kingella kingae is being increasingly recognized as the most frequent cause of bone and joint infection BJI in early childhood. The main objective of this prospective study is to report the frequency of Kingella Kingae in negative culture bone and joint pediatric infections, and to describe the clinical and biologic features of these children.

Methods: From December 2016 to June 2019, we selected all hospitalized patients with suspected BJI. When culture was negative on the fifth day, children under 10 years were subsequently included in the study, and PCR assay was performed systematically for researching K. kingae specific gene cpn60. Microbial culture and identification were made using standard bacteriological methods. The demographics, clinical, laboratory, radiographic and clinical features were reviewed from medical records.

Results: We enrolled 65 children with culture negative BJl, 46 of them having under 10 years old have been screened for the cpn60 gene. Thus, the gene encoding Kingella kingae was positive for $27 \mathrm{BJ}$ cases (58.7\%). The mean age of children was 3.02 years, $55.6 \%$ were aged 6 months- 4 years and $29.6 \%$ of them were aged $5-10$ years. The male to female ratio was 1.7 and 16 cases (59.26\%) occurred during the fall-winter period. The most frequent BJI type was septic arthritis (77.8\%) and the most affected sites were knee (51.9\%) and hip (37.0\%). We recorded a mild clinical picture with normal to mildly raised inflammatory markers. All patients had good clinical and functional outcomes, with no serious orthopedic sequelae..

Conclusion: $K$ kingae is an important pathogen of culture-negative $\mathrm{BJ}$ in Moroccan children. PCR testing should be performed in culture-negative cases of children not only in the typical age range of 6 months to 4 years. When implemented in the routine clinical microbiology laboratory, a specific K. kingae PCR assay can provide a better diagnostic performance of BJl.
\end{abstract}

Keywords: Kingella kingae, Bone and joint infection, Negative culture, Morocco

\footnotetext{
*Correspondence: bouchra.oumokhtar@usmba.ac.ma

'Laboratoire Pathologie Humaine Biomédecine et Environnement, Faculté de Médecine et de Pharmacie de Fès (FMPF), Université Sidi Mohammed Ben Abdellah (USMBA), Fès, Morocco

Full list of author information is available at the end of the article
}

(c) The Author(s). 2021 Open Access This article is licensed under a Creative Commons Attribution 4.0 International License, which permits use, sharing, adaptation, distribution and reproduction in any medium or format, as long as you give appropriate credit to the original author(s) and the source, provide a link to the Creative Commons licence, and indicate if changes were made. The images or other third party material in this article are included in the article's Creative Commons licence, unless indicated otherwise in a credit line to the material. If material is not included in the article's Creative Commons licence and your intended use is not permitted by statutory regulation or exceeds the permitted use, you will need to obtain permission directly from the copyright holder. To view a copy of this licence, visit http://creativecommons.org/licenses/by/4.0/ The Creative Commons Public Domain Dedication waiver (http://creativecommons.org/publicdomain/zero/1.0/) applies to the data made available in this article, unless otherwise stated in a credit line to the data. 


\section{Background}

Bone and joint infections (BJI) in children present a significant clinical challenge that could lead to life-long disability and death if not treated urgently [1]. Until recently, Staphylococcus aureus was considered the most common microorganism responsible for these infections. However, the progress of molecular diagnostic methods improved the detection of Kingella kingae from bone and joint samples. Currently, this bacteria is recognized in several countries, as the most frequent cause of BJIs especially among children aged between 6 and 48 months old. Affected children often have few signs and symptoms, and generally, clinical, radiological manifestations and biological inflammatory response to $K$. kingae BJI are mild to moderate [2].

$K$. kingae is typically a frequent component of the oropharyngeal microbiota of healthy young children. This asymptomatic colonization facilitates transmission between children notably through respiratory secretions [2]. Thus, the colonized epithelium is the gateway for the bacterium into the bloodstream where it disseminates to distant sites including bones, joints or endocardium. Ceroni et al. have reported a great risk of BJI with oropharyngeal carriage of $K$. kingae notably in children $<4$ years of age, corresponding to the period of maximal oropharyngeal colonization. After 4 years, the carry rate decreases significantly [3].

Using culture-based diagnostic methods in our hospital, we frequently observe negative cultures in pediatric BJI, and actually, we have no local data on the involvement of $\mathrm{K} . \mathrm{K}$ in this infection type. It was reported that K. kingae represented $30.8 \%(711 / 2308)$ of pediatric cases with culture and/or PCR proven musculoskeletal infections [4]. Recently, improvement of nucleic acid amplification assay targeting either the $r t x$ operon or the groEL gene (also known as cpn60) allowed a further increase in the diagnostic capability for pediatric K. kingae BJI [5].

$K$. kingae infections have been reported mainly in the countries of the developed world whereas reports from the developing world are still scarce, owing to the high charge of PCR technologies required to detect the bacteria [6]. In Morocco and to the best of our knowledge, no studies have been published on K. kingae BJI. Therefore, the objective of this study was to investigate the frequency of pediatric $K$. Kingae BJI diagnosed in negative culture by PCR, and also to describe the clinical and biologic features of patients.

\section{Methods}

\section{Study design and setting}

From December 2016 to June 2019, we selected all patients with suspected BJI hospitalized in Pediatric Orthopedic Traumatology Ward (Hassan II UH, Fez). This surgical department has 28 beds, providing medicalsurgical treatment for pediatric orthopedic disorders and also diseases of the musculoskeletal system. It's the only tertiary care facility of its kind in Fez, a city with an estimated population of approximately 1.5 million inhabitants.

Patients were subsequently included in the study when the culture was negative on the fifth day, and we performed PCR assay systematically for researching $K$. kingae specific gene. Two types of infection were distinguished: septic arthritis and bone infections, including osteitis and osteomyelitis. We excluded patients above 10 years old and those with osteosynthesis material.

This study was approved by local Joint Research Ethics Committee. Informed consent was obtained from each parent's patient before inclusion.

\section{Sampling and bacterial analysis}

Blood samples, joint fluid or bone aspirate were shipped to the laboratory immediately after sampling. Each specimen was divided into two parts; the first one was inoculated into the rich medias: chocolate agar (PVX, BioMerieux ${ }^{\oplus}$ ) and blood agar (COS, BioMerieux ${ }^{\oplus}$ ) and then incubated at $37^{\circ} \mathrm{C}$ for $24 \mathrm{~h}$ in aerobic and anaerobic conditions. The second one was inoculated into blood culture bottles (BD BACTEC ${ }^{\oplus}$ ) and incubated using a BD BACTEC ${ }^{\mathrm{\tau м}}$ instrumented blood culture system for 10 days. Isolates were identified using standard methods and confirmed by Api System (BioMerieux ${ }^{\oplus}$, Marcy l'Etoile, France).

\section{Molecular analysis}

$K$ kingae PCR testing was performed using residual bacterial culture-negative samples. These residual samples were stored at $-80^{\circ} \mathrm{C}$ until used for DNA extraction. The total DNA was extracted from specimens by using the PrepMan ${ }^{\mathrm{TM}}$ Ultra DNA extraction kit (Applied biosystems ${ }^{\oplus}$ ) according to the instruction. Then, the DNA concentration was measured by a NanoDrop 1000 spectrophotometer.

The DNA extracted were screened by PCR assay protocol targets the primers shown to be specific for the cpn60 gene (also known as groEL), which encode the Cpn60 chaperone protein: $K k F\left(5^{\prime}\right.$ - CTT-GCG-AAACAT-ACG-AGC-AA - 3') and KkR (5' - CCA-AACCAG-CGA-TTT-GTT-TT - 3'). The amplification conditions were described previously [7].

DNA $K$. kingae positive control was given graciously by Pr. Philippe Lanotte (CHRU de Tours). PCR products were detected on 1\% agarose gel (FMC Bioproduct, Rockland, ME) after ethidium bromide staining, UV illumination and photographed by an Olympus digital camera (Olympus Soft Imaging Solutions GmbH, Münster, Germany). 


\section{Data analysis}

The socio-demographic characteristics of patients were collected prospectively using a standard written questionnaire. Clinical data were recorded from the medical record of each patient, and the following data were collected: clinical and laboratory parameters at presentation; imaging studies; surgical procedures; duration of hospitalization; antibiotic treatment; complication and sequelae. The biologic evaluation comprised peripheral white cell counts (WBC), C-reactive protein levels (CRP), and erythrocyte sedimentation rate (ESR).

Three categories of patients have been defined. The first group of young infants under 6 months corresponds to the end of maternally derived immunity. The second group of older infants comprises children from six to 48 months, where $K$ kingae is recognized as the primarily affecting agent of this age. The third group includes juveniles over 4 years old and up to 10 years.

Results for quantitative variables were presented as mean \pm standard deviation and for qualitative variables as number (percentage). Identification of potential risk factors associated with the infection by $K$. kingae was performed as appropriate. Chi-square test and Fisher's exact test were used to establish a significant association between patients with $K$. kingae BJI and those with no $k$. kingae diagnosed. The $P<0.05$ was deemed as statistically significant. Data were analyzed using SPSS software version 20 (IBM Corporation, Chicago, IL, USA).

\section{Results}

We recorded 110 children diagnosed with BJI, 72 boys and 38 girls with ages ranging from 20 days to 15 years old. Bacterial culture from the joint, pus samples or bone tissue proved positive in $40.9 \%$ (45/110) among which $53.3 \%(24 / 45)$ were from septic arthritis, $31.1 \%(14 / 45)$ and $15.6 \%(7 / 45)$ respectively from osteomyelitis and multifocal abscess. Staphylococcus aureus methicillinsensitive (except one isolate) was the most common causative organism identified (82.2\%, 37 of 45). However, $K$. kingae was not revealed by the blood culture system despite the majority of BJI cases had not received antibiotics before surgical specimen.

Moreover, we showed that $59.1 \%$ of cases (65 of 110) were culture negative. Among them, the most frequent BJI types were septic arthritis (75.4\%) diagnosed following standard criteria adopted routinely in the ward, notably temperature above than $38.3^{\circ} \mathrm{C}$ and pain in the suspected joint that which got worse with notion, swelling in the suspected joint or systemic symptoms. Next comes osteomyelitis (18.5\%), and spondylodiscitis (3.1\%). The most frequent sites of infection were knee and hip by 45.7 and $34.8 \%$ respectively.

For this study we selected only patients having under 10 years old $(n=46)$. The mean age of children managed was $4.55 \pm 3.11$ years, $29(63.0 \%)$ were boys and almost one-third of the patients were from rural settings. The mean temperature at admission was $38.53{ }^{\circ} \mathrm{C}$, The mean of CRP value was $94.78 \mathrm{mg} / \mathrm{L}$, and the mean ESR was $55.33 \pm 32.52 \mathrm{~mm} / \mathrm{H}$.

All cultures were negative despite inoculating blood culture vials with joint fluid samples. However, 37 samples were positive for cpn60, the gene encoding $K$. kingae detected by PCR (58.7\%). The mean age of $K$. kingae positive patients was $3.02 \pm 2.50$ years; ranging from $<1$ month to 8 years. The male to female ratio was 1.7 . Analyzing our results by age group showed that $14.8 \%(4 / 27)$ were under 6 months, and 2 of them were neonates, $55.6 \%$ of children were aged 6 to 48 months and $29.6 \%$ of infants were 48-96 months. K. kingae prevalence rate differs significantly between the population age groups $(P=0.009$; Table 1). Out of 27 K. kingae BJI, 16 (59.26\%) occurred during the fall-winter period. The time elapsed between the onset of symptoms and hospitalization was 10.4 days (SD 18.25 days).

In addition, $77.8 \%$ of the $K$. kingae-positive children group had septic arthritis, $14.8 \%$ had osteomyelitis and $7.4 \%$ had spondylodiscitis. More than half had an infection of the knee joint, $37.0 \%$ of the hip, and $11.1 \%$ of the femur. No infection was shown in the shoulder, humerus, forearm, wrist and foot (Table 1).

Generally, we recorded a mild clinical picture with normal to mildly raised inflammatory markers. The median temperature reported at admission was $38.60{ }^{\circ} \mathrm{C}$ and the mean temperature was $38.62^{\circ} \mathrm{C}$. The mean CRP value was $96.31 \mathrm{mg} / \mathrm{L}, 85.2 \%$ of patients having values > $20 \mathrm{mg} / \mathrm{L}$ and $77.8 \%>50 \mathrm{mg} / \mathrm{L}$. The mean Erythrocyte sedimentation rate was $54.50 \pm 35.65(\mathrm{~mm} / \mathrm{H})$; and the mean WBC rate was $13,429.9 \pm 6063.9 / \mathrm{mm}^{3}$. Clinical features of the children with negative and positive $K$. kingae BJI are summarized in Table 2.

Two of the children found to be PCR-positive for $K$. kingae have prior antibiotic therapy before collection of joint fluid samples for culture. The average hospital stay was 11.17 days. Only one patient with $K$. kingae positive tibial acute osteomyelitis had a complication, consisting of metaphyseal pus collection that necessitates multiples drainages. This patient had evolution to chronic osteitis with a lesion of the proximal tibial growth plate. We reported good clinical and functional outcomes following antibiotic treatment for all the rest patients.

\section{Discussion}

Bone and joint infection BJI is a potentially devastating disorder with a high incidence of severe and long-lasting sequelae, particularly in growing children. Improving patient management requires determining the causative agent to prevent the risk of long-term disabilities [8]. Staphylococcus aureus is the most common pathogen in 
Table 1 Data of patients with negative and positive $K$. kingae BJl cases

\begin{tabular}{|c|c|c|c|}
\hline Characteristics & K. kingae- negative cases $N=19$ & K. kingae-positive cases $N=27(58.7 \%)$ & $P$-value \\
\hline \multicolumn{4}{|l|}{ Demographics: } \\
\hline \multicolumn{4}{|l|}{ Age (years) } \\
\hline$<6$ months & $0(0)$ & $4(14.8)$ & \multirow[t]{3}{*}{0.009} \\
\hline 6-59 months & $5(26.3)$ & $15(55.6)$ & \\
\hline $5 y-10 y$ & $14(73.7)$ & $8(29.6)$ & \\
\hline \multicolumn{4}{|l|}{ Gender } \\
\hline Male & $12(63.2)$ & $17(63.0)$ & \multirow[t]{2}{*}{0.618} \\
\hline Female & $7(36.8)$ & $10(37.0)$ & \\
\hline \multicolumn{4}{|l|}{ Origin } \\
\hline Rural & $8(42.1)$ & $8(29.6)$ & \multirow[t]{2}{*}{0.287} \\
\hline Urban & $11(57.9)$ & $19(70.4)$ & \\
\hline \multicolumn{4}{|l|}{ Admission features: } \\
\hline \multicolumn{4}{|l|}{ Type of the infection } \\
\hline Septic arthritis & $15(78.9)$ & $21(77.8)$ & \multirow[t]{4}{*}{0.417} \\
\hline Osteomyelitis & $3(15.8)$ & $4(14.8)$ & \\
\hline Multifocal abscesses & $1(5.3)$ & $0(0)$ & \\
\hline Spondilodiscitis & $0(0)$ & $2(7.4)$ & \\
\hline \multicolumn{4}{|l|}{ Site of the infection } \\
\hline Single & $17(89.5)$ & $24(88.9)$ & \multirow[t]{2}{*}{0.448} \\
\hline Multiple & $2(10.5)$ & $3(11.1)$ & \\
\hline \multicolumn{4}{|l|}{ Location of limb infection } \\
\hline Upper & $1(5.3)$ & $3(11.1)$ & \multirow[t]{2}{*}{0.632} \\
\hline Lower & $18(94.7)$ & $24(88.9)$ & \\
\hline \multicolumn{4}{|l|}{ Limb affected } \\
\hline Hip & $6(31.6)$ & $10(37.0)$ & 0.475 \\
\hline Femur & $2(10.5)$ & $3(11.1)$ & 0.667 \\
\hline Knee & $7(36.8)$ & $14(51.9)$ & 0.241 \\
\hline \multicolumn{4}{|l|}{ Diagnosis on admission } \\
\hline Magnetic resonance imaging & $1(5.3)$ & $2(7.4)$ & 0.632 \\
\hline Pathologic ultrasound & $8(42.1)$ & $14(51.9)$ & 0.191 \\
\hline Joint effusion & $5(31.3)$ & $5(26.3)$ & 0.519 \\
\hline Soft tissue infiltration & $5(31.3)$ & $11(57.9)$ & 0.108 \\
\hline soft tissue abscesses & $1(6.3)$ & $6(31.6)$ & 0.062 \\
\hline Abnormal plain radiograph & $3(15.8)$ & $1(3.7)$ & 0.208 \\
\hline Purulent discharge & $6(31.6)$ & $3(11.1)$ & 0.085 \\
\hline
\end{tabular}

acute BJI, being identified in 70 to $90 \%$ of culture positive cases. In our study, Staphylococcus aureus was the main causative agent of infections recovered at $82.2 \%$ on a positive culture. However, we showed that $59.1 \%$ of samples (65 of 110) remained culture negative. Dodwell has reported that no organism is identified in up to $55 \%$ of pediatric BJI cases, even if appropriate samples had been obtained [9].

Recently, molecular diagnosis has significantly increased positive results, especially among $K$. kingae infections. In countries where $K$. kingae-specific realtime PCR (RT-PCR) assays are routinely employed this organism is recognized as the main agent of BJI in young children $[10,11]$. In fact, to date, little is known on the epidemiology of $K$. kingae BJIs in children in the African continent. To the best of our knowledge, we report the first study of $K$. kingae BJI among Moroccan children population. We revealed that $K$. kingae was the leading cause $(58.7 \%)$ of culture-negative BJIs in young children. This rate are higher than 36.07 and $45 \%$ reported in 
Table 2 Clinical features of patients with negative and positive $K$. kingae BJ cases

\begin{tabular}{|c|c|c|c|}
\hline Clinical features on admission & K. kingae- negative cases $N=19$ & K. kingae-positive cases $N=27$ (58.7\%) & $P$-value \\
\hline Fever $(\geq 38)^{\circ} \mathrm{C}$ & $12(63.2)$ & $19(70.4)$ & 0.421 \\
\hline Initial value, mean $( \pm S D)$ & $38.73 \pm 0.64$ & $38.62 \pm 0.53$ & 0.625 \\
\hline \multicolumn{4}{|l|}{ CRP (mg/l) } \\
\hline Initial value, mean ( \pm SD) & $92.61 \pm 79.95$ & $96.31 \pm 57.07$ & 0.855 \\
\hline Elevated CRP on admission & $17(89.5)$ & $23(85.2)$ & 0.516 \\
\hline \multicolumn{4}{|l|}{$\operatorname{ESR}(\mathrm{mm} / \mathrm{H})$} \\
\hline Initial value, mean $( \pm S D)$ & $56.44 \pm 29.90$ & $54.50 \pm 35.65$ & 0.896 \\
\hline Elevated ESR on admission & $8(42.1)$ & $12(44.4)$ & 0.484 \\
\hline \multicolumn{4}{|l|}{$\mathrm{WBC} / \mathrm{mm}^{3}$} \\
\hline Initial value, mean ( \pm SD) & $13,554.7 \pm 4596.6$ & $13,429.9 \pm 6063.9$ & 0.940 \\
\hline Elevated WBC on admission & $15(78.9)$ & $21(77.8)$ & 0.925 \\
\hline Arthrotomy & $8(42.1)$ & $19(70.4)$ & 0.053 \\
\hline Hospital stay, days, mean ( \pm SD) & $9.64 \pm 5.80$ & $11.17 \pm 7.27$ & 0.508 \\
\hline
\end{tabular}

France $[10,12]$, or $25.93 \%$ in Ottawa [13]. K. kingae infections seem to be closely correlated to children's ages. In a meta-analysis, Wong et al. reported $47.6 \%$ of patients under 48 months of age were diagnosed with $K$. kingae BJI infections [4]. In this study, $55.6 \%$ of children were aged 6 to 48 months, and $14.8 \%$ (4/ 27) were under 6 months, 2 of them were aged $<1$ month, with no history of trauma or unusual infections, and no warning signs of immunosuppression were found. Also, 29.6\% (8/27) of children were over 48 months (5 years and older (5-8.2 years), close contact with younger children or siblings is very likely, which may explain this results. This finding is not corroborating with the classic representation of $K$. kingae BJI, which occurs in children between 6 months and 4 years old. Shahrestani et al. [14] and more recently, Ceroni et al. have established the possibility of $K$. kingae osteoarticular infections in older immunocompetent children [15]. Also, we diagnosed a large proportion of $K$. kingae patients during the fall to winter season. Wong et al. have reported that this seasonal variation is likely associated with respiratory viral infections and stomatitis, allowing for passage of the colonizing agent through the breached epithelium [4]. Concerning the site of infection, K. kingae are responsible of septic arthritis with variable of 45 to $69 \%$ $[8,10,12,16]$. We recorded 21 cases of septic arthritis (77.8\%), 4 osteomyelitis (14.8\%) and 2 spondylodiscitis. Similarly to published data, our finding confirms that infection tends to affect the lower extremity the most, and the knee is often involved [16], and almost all cases present a moderate clinical picture with normal to slightly elevated inflammatory markers [6]. in fact, chronic osteomyelitis and orthopedic sequelae seem to be uncommon in $K$. kingae BJI in young children [2, 8].

Unfortunately, we were unable to establish an antimicrobial susceptibility pattern, because all culture was negative despite inoculation into blood culture vials. It has been recognized that $K$. kingae is most often sensitive to many classes of antibiotics (beta-lactams, macrolides, aminoglycosides, fluoroquinolones, tetracyclins) [13]. For managing BJI in our ward, empirical antibiotic therapy is started with intravenous antibiotics (clavulanic-acid/amoxicillin and gentamycin), and after discharge, treatment continues orally by 10 days to 3 weeks according to the judgment of the attending physician. In the case of documented $K$. kingae BJI, the antibiotic treatment consisted of oral clavulanic-acid/amoxicillin for 14 to 21 days. Several authors have suggested switching to trimethoprim-sulfamethoxazole once $K$ kingae infection was identified $[6,14]$.

For PCR analysis, we targeted the groEL gene (also known cpn60), a housekeeping gene encoding a chaperone protein recognized as a universal bacterial marker [17]. Recently, El Houmani et al. provided evidence that kkgroEL gene could discriminate even $K$. kingae from $K$. negevensis [14].

Our study had several limits, the first of which was the one site of study and the small sample size. Despite this, we recorded $58.7 \% K$. kingae $\mathrm{BJI}$, providing evidence that $K$. kingae is circulating in our region as well. Also, we could not define antimicrobial susceptibility patterns in our region because no $K$. Kingae was growing in culture media even we inoculated joint samples into aerobic blood vials. Further studies are required to determine the prevalence of $K$. kingae carriage and BJI in other Moroccan sites. 


\section{Conclusion}

$K$ kingae is an important pathogen of culture-negative BJI in Moroccan children. PCR testing should be performed in culture-negative cases of children not only in the typical age range of 6 months to 4 years. When implemented in the routine clinical microbiology laboratory, a specific $K$. kingae PCR assay can provide a better diagnostic performance of BJI.

\section{Acknowledgements}

Pr. Philippe Lanotte from bacteriology unit, Service de BactériologieVirologie-Hygiène Hospitalière, Hôpital Bretonneau - CHRU de Tours, to provide Kingella kingae DNA as a positive control.

\section{Authors' contributions}

All authors have made substantial contributions to conception, to design, to acquisition of data, to analysis and to interpretation of data; All authors have been involved in drafting the manuscript or revising it critically for important intellectual content; All authors have given final approval of the version to be published. Each author has participated sufficiently in the work to take public responsibility for appropriate portions of the content. All authors have agreed to be accountable for all aspects of the work in ensuring that questions related to the accuracy or integrity of any part of the work are appropriately investigated and resolved. All authors read and approved the final manuscript.

\section{Funding}

Not applicable.

\section{Availability of data and materials}

The datasets used and/or analyzed during the current study are available from the corresponding author on reasonable request.

\section{Declarations}

\section{Ethics approval and consent to participate}

This study was approved by the Joint Research Ethics Committee of Medical School and the Hassan II University Hospital (Fez, Morocco). Written information about the nature of the experimental procedures was given to the parents of the patients, who provided the informed consent for participation of their children. All protocol was performed in accordance with the relevant guidelines and regulations/Declaration of Helsinki.

\section{Consent for publication}

Not applicable.

\section{Competing interests}

The authors declare that they have no competing interests.

\section{Author details}

'Laboratoire Pathologie Humaine Biomédecine et Environnement, Faculté de Médecine et de Pharmacie de Fès (FMPF), Université Sidi Mohammed Ben Abdellah (USMBA), Fès, Morocco. ${ }^{2}$ Service de traumato-orthopédie pédiatrique, CHU Hassan II. Laboratoire Pathologie humaine Biomédecine et Environnement. Faculté de Médecine et de Pharmacie, Université Sidi Mohamemd Ben Abdellah, Fès, Morocco. ${ }^{3}$ Laboratoire Epidémiologie, Recherche Clinique et Santé Communautaire. Faculté de Médecine et de Pharmacie, Université Sidi Mohammed Ben Abdellah, Fès, Morocco. ${ }^{4}$ Laboratoire Centrale d'analyses médicales, CHU Hassan II. Faculté de Médecine et de Pharmacie, Université Sidi Mohammed Ben Abdellah, Fès, Morocco.

Received: 21 March 2021 Accepted: 14 June 2021

Published online: 20 July 2021

\section{References}

1. Cherkaoui A, Ceroni D, Emonet S, Lefevre Y, Schrenzel J. Molecular diagnosis of Kingella kingae osteoarticular infections by specific real-time PCR assay. J Med Microbiol. 2009;58(1):65-8. https://doi.org/10.1099/jmm.0.47707-0.
2. Yagupsky P, Porsch E, St Geme JW. Kingella kingae: an emerging pathogen in young children. PEDIATRICS. 2011;127(3):557-65. https://doi.org/10.1542/ peds.2010-1867.

3. Ceroni D, Dubois-Ferriere V, Cherkaoui A, Gesuele R, Combescure C, Lamah $L$, et al. Detection of Kingella kingae Osteoarticular infections in children by oropharyngeal swab PCR. PEDIATRICS. 2013 Jan 1;131(1):e230-5. https://doi. org/10.1542/peds.2012-0810

4. Wong M, Williams N, Cooper C. Systematic review of Kingella kingae musculoskeletal infection in children: epidemiology, Impact and Management Strategies. PHMT. 2020;11:73-84.

5. El Houmami N, Bzdrenga J, Durand GA, Minodier P, Seligmann H, Prudent E, et al. Molecular tests that target the RTX locus do not distinguish between Kingella kingae and the recently described Kingella negevensis species. McAdam AJ, editor. J Clin Microbiol. 2017;55(10):3113-22. https://doi.org/1 0.1128/JCM.00736-17.

6. Yagupsky P. Kingella kingae: carriage, transmission, and disease. Clin Microbiol Rev. 2015 Jan;28(1):54-79. https://doi.org/10.1128/CMR.00028-14.

7. Baticle E, de Courtivron B, Baty G, Holstein A, Morange V, Mereghetti L, et al. Infections ostéo-articulaires pédiatriques à Kingella kingae de 1995 à 2006 au CHRU de Tours. Ann Biol Clin. 2008;66:5.

8. Yagupsky P. Kingella kingae: from medical rarity to an emerging paediatric pathogen. Lancet Infect Dis. 2004;4(6):358-67. https://doi.org/10.1016/S14 73-3099(04)01046-1.

9. Dodwell ER. Osteomyelitis and septic arthritis in children: current concepts. Curr Opin Pediatr. 2013;25(1):58-63. https://doi.org/10.1097/MOP.0b013e32 $835 c 2 b 42$.

10. Ilharreborde B, Bidet P, Lorrot M, Even J, Mariani-Kurkdjian P, Liguori S, et al. New real-time PCR-based method for Kingella kingae DNA detection: application to samples collected from 89 children with acute arthritis. J Clin Microbiol. 2009;47(6):1837-41. https://doi.org/10.1128/JCM.00144-09.

11. El Houmami N, Fournier P-E, Ceroni D. Targeting the Kingella Kingae groEL gene is a reliable method for the molecular diagnosis of $K$. Kingae infection and carriage. J Paediatr Child Health. 2017;53(10):1030-1. https://doi.org/1 $0.1111 /$ jpc.13672.

12. Chometon S, Benito Y, Chaker M, Boisset S, Ploton C, Bérard J, et al. Specific Real-Time Polymerase Chain Reaction Places Kingella kingae as the Most Common Cause of Osteoarticular Infections in Young Children. Pediatr Infect Dis J. 2007;26(5):377-81. https://doi.org/10.1097/01.inf.0000259954. 88139.f4.

13. Slinger R, Moldovan I, Bowes J, Chan F. Polymerase chain reaction detection of Kingella kingae in children with culture-negative septic arthritis in eastern Ontario. Paediatr Child Health. 2016;21(2):79-82. https://doi.org/10.1093/ pch/21.2.79.

14. Shahrestani S, Evans A, Tekippe EM, Copley LAB. Kingella kingae septic arthritis in an older-than-expected child. J Pediatr Infect Dis Soc. 2019;8(1): 83-6. https://doi.org/10.1093/jpids/piy063.

15. Ceroni D, Coulin B, Vendeuvre T, Morello V, De Marco G, Dayer R, et al. Should we investigate Osteoarticular infections for Kingella kingae in olderthan-expected immunocompetent children? Pediatr Infect Dis J. 2020;39(5): e57-8. https://doi.org/10.1097//NF.0000000000002613.

16. Aupiais C, Ilharreborde B, Doit C, Blachier A, Desmarest M, Job-Deslandre C, et al. Aetiology of arthritis in hospitalised children: an observational study. Arch Dis Child. 2015;100(8):742-7. https://doi.org/10.1136/archdischild-2 014-307490.

17. Links MG, Dumonceaux TJ, Hemmingsen SM, Hill JE. The Chaperonin-60 Universal Target Is a Barcode for Bacteria That Enables De Novo Assembly of Metagenomic Sequence Data. Neufeld J, editor. PLoS One. 2012;7(11): e49755

\section{Publisher's Note}

Springer Nature remains neutral with regard to jurisdictional claims in published maps and institutional affiliations. 